\title{
X-ray confirmation of the intermediate polar HT Cam ${ }^{\star}$
}

\author{
D. de Martino ${ }^{1}$, G. Matt ${ }^{2}$, K. Mukai ${ }^{3}$, J.-M. Bonnet-Bidaud ${ }^{4}$, B. T. Gänsicke ${ }^{5}$, \\ J. M. Gonzalez Perez ${ }^{6}$, F. Haberl ${ }^{7}$, M. Mouchet ${ }^{8}$, and J.-E. Solheim ${ }^{9}$
}

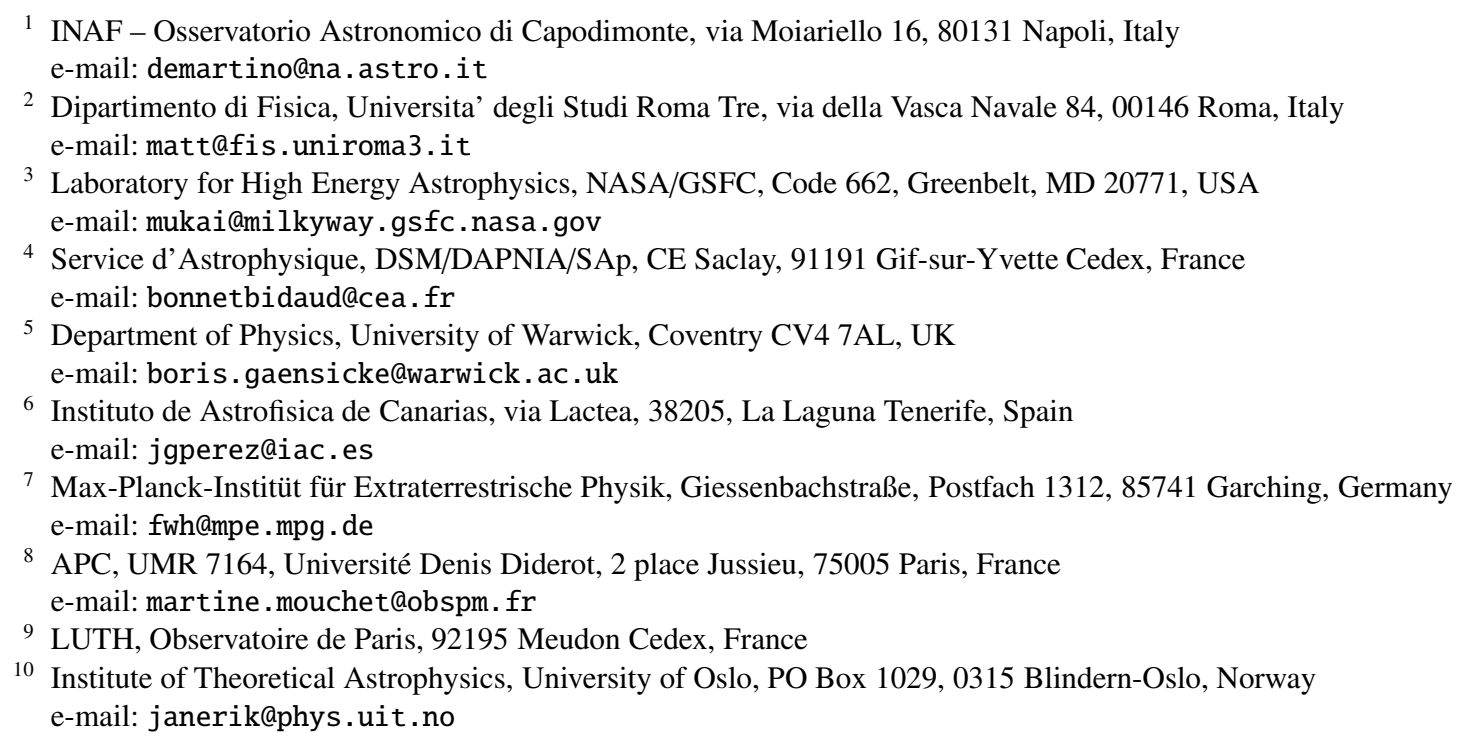

Received 25 January 2005 / Accepted 19 March 2005

\begin{abstract}
We report on the first pointed X-ray observations with XMM-Newton and RXTE satellites of the X-ray source RX J0757.0+6306 = HT Cam. We detect a strong $515 \mathrm{~s} \mathrm{X-ray} \mathrm{modulation} \mathrm{confirming} \mathrm{the} \mathrm{optical} \mathrm{photometric} \mathrm{period} \mathrm{found} \mathrm{in}$ 1998, which definitively assigns this source to the intermediate polar class of magnetic cataclysmic variables. The lack of orbital sidebands in the X-rays indicates that the X-ray period is the spin period of the accreting white dwarf. Simultaneous ultraviolet and optical $B$-band photometry acquired with the XMM-Newton Optical Monitor and coordinated optical UBVRI photometric data acquired at the Nordic Optical Telescope (La Palma) show that the optical pulse is in phase with the X-rays and hence originates in the magnetically-confined accretion flow. The lack of ultraviolet spin modulation suggests that accretion-induced heating on the white dwarf surface is not important in this source. Spectral analyses of XMM-Newton EPIC and RGS data show that HT Cam has a multi-temperature spectrum and, contrary to most intermediate polars, it does not suffer from strong absorption. With its 86 min orbital period, HT Cam is the third confirmed system of this class below the 2-3 h period gap accreting at a low rate.
\end{abstract}

Key words. stars: binaries: close - stars: individual: HT Cam - stars: novae, cataclysmic variables

\section{Introduction}

RX J0757.0+6306 = HT Cam (henceforth HT Cam) was identified as a short orbital period $(81 \mathrm{~min})$ cataclysmic variable (CV) and proposed as an intermediate polar (IP) by Tovmassian et al. (1998) for its rapid photometric variability at a period of $8.5 \mathrm{~min}$. These periods were recently refined from spectroscopy $\left(P_{\text {orb }}=85.98 \mathrm{~min}\right)$ and from extensive photometry $\left(P_{\text {spin }}=\right.$ 515.06 s) by Kemp et al. (2002), further supporting the IP identification. Whether the short period pulse represents the spin

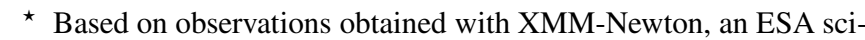
ence mission with instruments and contributions directly funded by ESA Member States and NASA. period of the accreting white dwarf (WD) or an orbital sideband could not be assessed with the optical photometry alone (see also Warner 1986, for the origin of optical pulsations in IPs). In IPs the accretion flow is channelled by the relatively strong ( $B \leq 5-10 \mathrm{MG}$ ) magnetic field of the WD onto its polar regions where a strong shock develops above the WD surface, below which the gas cools via thermal bremsstrahlung peaking in the hard X-ray regime. Hence, the signature of channelled accretion in these systems is a strong X-ray pulsation at the WD rotational period which could not be confirmed by the short ROSAT observation performed during the All Sky Survey on this source. HT Cam also occasionally displays short outbursts (Kemp et al. 2002; Ishioka et al. 2002), with a decline 
rate of $\sim 4$ mag day $^{-1}$, thus sharing this peculiarity with another short orbital period IP, EX Hya.

In the framework of a programme aiming at studying the X-ray properties of new magnetic CVs, we were granted XMM-Newton and RXTE observations during AO2 (PI: de Martino) and cycle 7 (PI: Mukai) respectively, which are described in Sect. 2. Furthermore, a coordinated optical multicolor photometric run at the Nordic Optical Telescope (NOT) (La Palma) was carried out simultaneously with the RXTE observation, also reported in Sect. 2. We present the X-ray and optical/UV timing analyses in Sects. 3 and 4 respectively and the X-ray spectral analysis is reported in Sect. 5. We discuss the implications of X-ray and optical results in Sect. 6 in terms of accretion parameters and evolutionary state.

\section{The observations}

\subsection{The XMM-Newton observation}

HTCam was observed with XMM-Newton satellite (Jansen et al. 2001) on 2003 March 24 (obsid: 0144840101) with the EPIC-PN (Strüder et al. 2001) and MOS (Turner et al. 2001) cameras operated in full frame mode with the medium filter for a net exposure time of $39.0 \mathrm{ks}$ and $39.8 \mathrm{ks}$ respectively. HT Cam was also observed with the Reflection Grating Spectrographs (RGS1 and RGS2) (den Herder et al. 2001) in spectroscopy mode with an exposure time of $40.5 \mathrm{ks}$ and with the Optical Monitor (OM) instrument (Mason et al. 2001) with the $U V W 2$ and $B$ filters covering the ranges 1800-2250 $\AA$ and 3900-4900 $\AA$ in imaging fast mode for a total exposure time of $17.5 \mathrm{ks}$ in each filter. A summary of the observations is reported in Table 1.

The standard pipeline processing data products were used. The EPIC light curves and spectra and the RGS spectra were extracted with the SAS 6.0 package retrieved from the ESA-VILSPA Science Center. EPIC light curves and spectra of HTCam were extracted from circular regions with a radius of $40^{\prime \prime}$ centred on the source, while background light curves and spectra were extracted from an offset circular region with radius of 90". Background subtracted EPIC net light curves were obtained taking into account the different areas of extraction regions. Only single and double pixel events with a zero quality flag were selected for the EPIC-PN data, while for EPIC-MOS cameras all valid patterns have been used (see XMM-Newton SAS User Guide: http: //xmm.vilspa.esa.es/external/ xmm_user_support/documentation/sas_usg/USG). The EPIC-PN and, at a less extent, the MOS data, are affected by a strong background activity and hence data were windowed in order to exclude the high background epochs. The extracted EPIC-PN and MOS spectra were rebinned to have a minimum of 25 counts in each bin to allow the use of the $\chi^{2}$ statistics. Ancillary response and redistribution matrix files were created using SAS meta-tasks arfgen and rmfgen respectively.

The RGS pipeline was run using the SAS meta-task rgsproc, and adopting the same temporal windowing used for the EPIC cameras to exclude high background intervals. RGS1 and RGS2 first order spectra have been rebinned to have a
Table 1. Summary of the observations of HT Cam.

\begin{tabular}{|c|c|c|c|c|}
\hline Telescope & Instrument & Date & UT (start) & Duration (s) \\
\hline \multirow[t]{13}{*}{ XMM-Newton } & EPIC PN & 2003 March 24 & $13: 21$ & 38997 \\
\hline & EPIC MOS & & $12: 58$ & 39810 \\
\hline & RGS & & $12: 58$ & 40523 \\
\hline & OM B & & $13: 08$ & 3499 \\
\hline & & & $14: 11$ & 3500 \\
\hline & & & $15: 15$ & 3498 \\
\hline & & & $16: 18$ & 3499 \\
\hline & & & $17: 22$ & 3499 \\
\hline & OM UVW2 & & $18: 26$ & 3500 \\
\hline & & & $19: 30$ & 3500 \\
\hline & & & $20: 34$ & 3498 \\
\hline & & & $21: 38$ & 3499 \\
\hline & & & $22: 41$ & 3500 \\
\hline \multirow[t]{2}{*}{$R X T E$} & PCA & 2002 Dec. 26 & $21: 57$ & 25000 \\
\hline & & 2002 Dec. 27 & $21: 51$ & 24000 \\
\hline \multirow[t]{5}{*}{$N O T$} & ALFOSC V & 2002 Dec. 26 & $20: 55$ & 12600 \\
\hline & ALFOSC B & 2002 Dec. 27 & $03: 15$ & 4440 \\
\hline & ALFOSC U & 2002 Dec. 27 & $20: 25$ & 11220 \\
\hline & ALFOSC R & 2002 Dec. 28 & $00: 46$ & 10920 \\
\hline & ALFOSC I & 2002 Dec. 28 & $04: 46$ & 5820 \\
\hline
\end{tabular}

minimum of 20 counts per bin. Ancillary and response files were also created for the RGS1 and RGS2 spectra.

OM background subtracted light curves, binned in $10 \mathrm{~s}$ intervals, produced by the standard processing pipeline were used for timing analysis. Average net count rates are $5.32 \mathrm{cts} \mathrm{s}^{-1}$ ( $B$ filter) and $0.326 \mathrm{cts} \mathrm{s}^{-1}$ ( $U V W 2$ filter). Using the zero points available at the VILSPA on-line documentation (http://xmm.vilspa.esa.es/external/ xmm_user_support /documentation /uhb/node75.html), the instrumental magnitudes are $B=17.2 \pm 0.5 \mathrm{mag}$ and $U V W 2=16.1 \pm 0.7 \mathrm{mag}$. Using Vega magnitude to flux conversion, these correspond to a flux of $8.09 \times$ $10^{-16} \mathrm{erg} \mathrm{cm}^{-2} \mathrm{~s}^{-1} \AA^{-1}$ in the 3900-4900 $\AA$ band and of $2.04 \times 10^{-15} \mathrm{erg} \mathrm{cm}^{-2} \mathrm{~s}^{-1} \AA^{-1}$ in the $1800-2250 \AA$ band. The $B$ band flux is then similar to that observed during quiescence.

\subsection{The RXTE data}

HT Cam was observed with RXTE/PCA instrument on 2002 December 26 and again on December 27. Although the PCA comprises 5 proportional counter units (PCUs), PCU0 suffers from a high background rate due to a leak in the propane layer, and PCUs 1, 3, and 4 are often switched off for instrumental reasons. We have therefore analyzed only the PCU2 data. After standard screening, good data were obtained for $25 \mathrm{ks}$ on the first observation and $24 \mathrm{ks}$ on the second. Background subtraction was performed using the "Faint" background model, with calibration file dated 2003 November 13. HTCam is detected with RXTE, however near the limit of background subtraction uncertainties. A spectral fit using the best-fit EPIC model (cf. Sect. 5) suggests a similar flux level as 

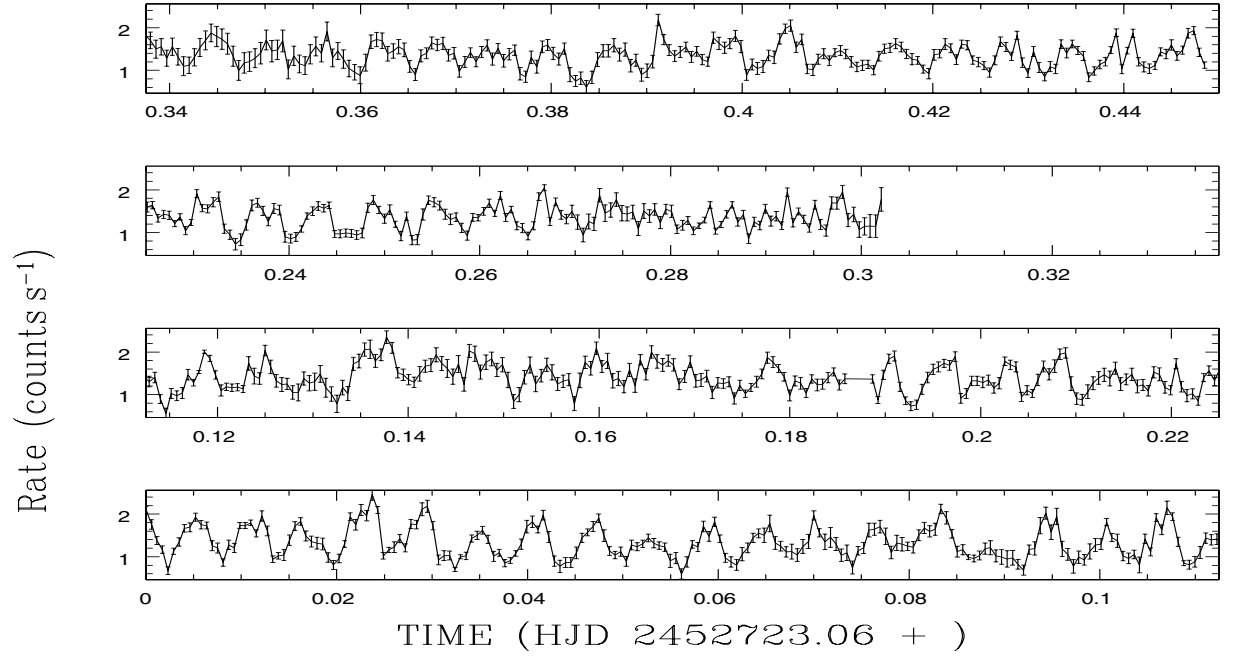

Fig. 1. EPIC-PN light curve in the $0.2-15 \mathrm{keV}$ range binned in $50 \mathrm{~s}$ intervals. High background epochs have been windowed as discussed in the text

during the XMM-Newton observations, although a systematic pattern in the residuals is present.

\subsection{The UBVRI photometry}

HT Cam was observed on 2002 December 26/27 and 27/28 at the NOT $2.56 \mathrm{~m}$ telescope (La Palma) equipped with ALFOSC camera operated in windowed fast photometric mode with $U B V R I$ filters simultaneously with the RXTE observations. Exposure times were such that the time resolution of CCD data acquisition (exposure, read-out and dead times) was of $60 \mathrm{~s}(U), 30 \mathrm{~s}(B$ and $V)$ and $20 \mathrm{~s}(R$ and $I)$. The details of the observations are reported in Table 1. During the first night the photometric conditions were not good due to strong wind especially during the second part of the night, thus only a short coverage with the $B$ band filter could be performed. The processing of the CCD images was performed using rtp and rtcorr softwares available at the NOT using aperture photometry, which include flat field correction, sky subtraction, differential photometry with several comparison stars in the field, extinction correction and data normalisation by least square fitting. For all filters the same comparison star has been used to analyse the light curves.

\section{X-ray timing analysis}

\subsection{The XMM-Newton light curves and power spectra}

EPIC PN and MOS light curves in the $0.2-15 \mathrm{keV}$ range were extracted with a $50 \mathrm{~s}$ time resolution and in Fig. 1 we report the PN net light curve only as they all show a similar behaviour. A clear indication of a variability on time scales of several minutes is found. The time series have been Fourier analysed to detect periodic signals as shown in Fig. 2. A strong peak is found at $168 \mathrm{day}^{-1}$ with no other significant peak close-by. The CLEAN algorithm (Roberts et al. 1987) was also applied to remove windowing effects introduced by the exclusions of high background epochs. This enables us to definitively exclude the presence of orbital sidebands. Weak signals at the

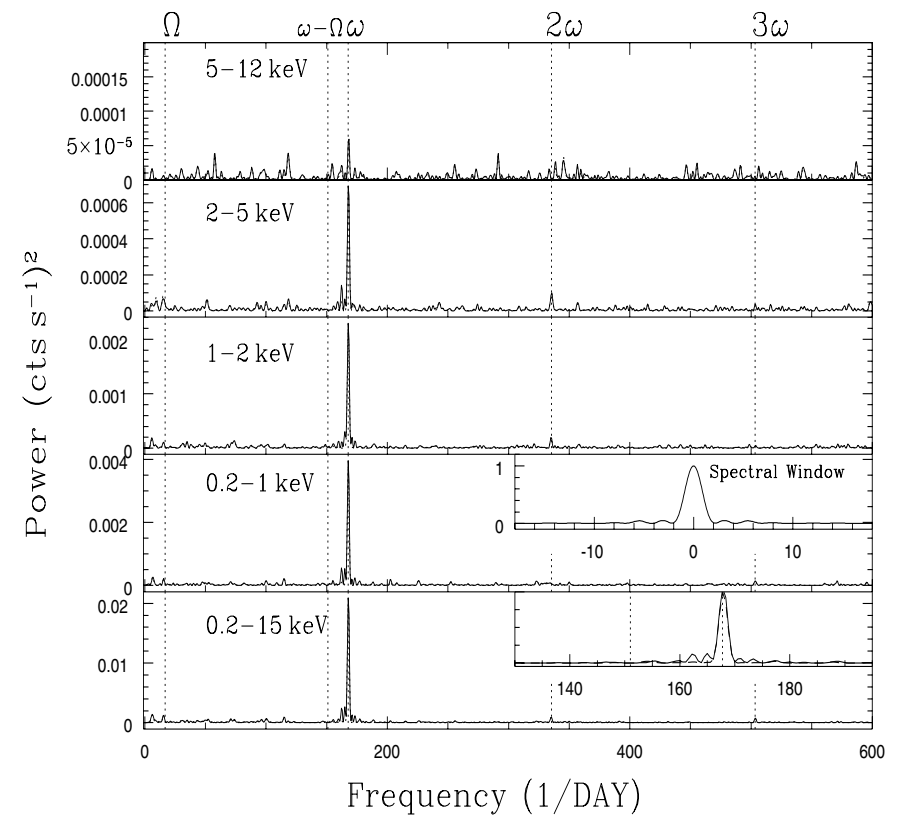

Fig. 2. EPIC-PN power spectra in selected energy ranges. From bottom to top: $0.2-15 \mathrm{keV}, 0.2-1 \mathrm{keV}, 1-2 \mathrm{keV}, 2-5 \mathrm{keV}$ and $5-12 \mathrm{keV}$. The spin $(\omega)$ and harmonics, the beat $(\omega-\Omega)$ and the orbital $(\Omega)$ frequencies are marked with vertical dotted lines. An enlargment around the spin frequency is shown in the inserted bottom panel where the DFT (solid line) together with the CLEANED spectrum (dashed line) are shown. The spectral window is also shown.

first and second harmonic are also observed. No significant peak is found at the orbital frequency. A sinusoidal fit to the PN and MOS light curves gives $\omega=167.90 \pm 0.05$ day $^{-1}$ for the PN, $168.04 \pm 0.07 \mathrm{day}^{-1}$ for MOS1 and $167.88 \pm$ $0.08 \mathrm{day}^{-1}$ for MOS2. The inclusion of additional sinusoids, accounting for the first and second harmonics does not improve the fit quality. We then adopt the EPIC PN period determination: $P_{\text {spin }}=514.59 \pm 0.15 \mathrm{~s}$ which agrees within $3 \sigma$ with the refined optical pulse period found by Kemp et al. (2002). We hence determine the following ephemeris from 

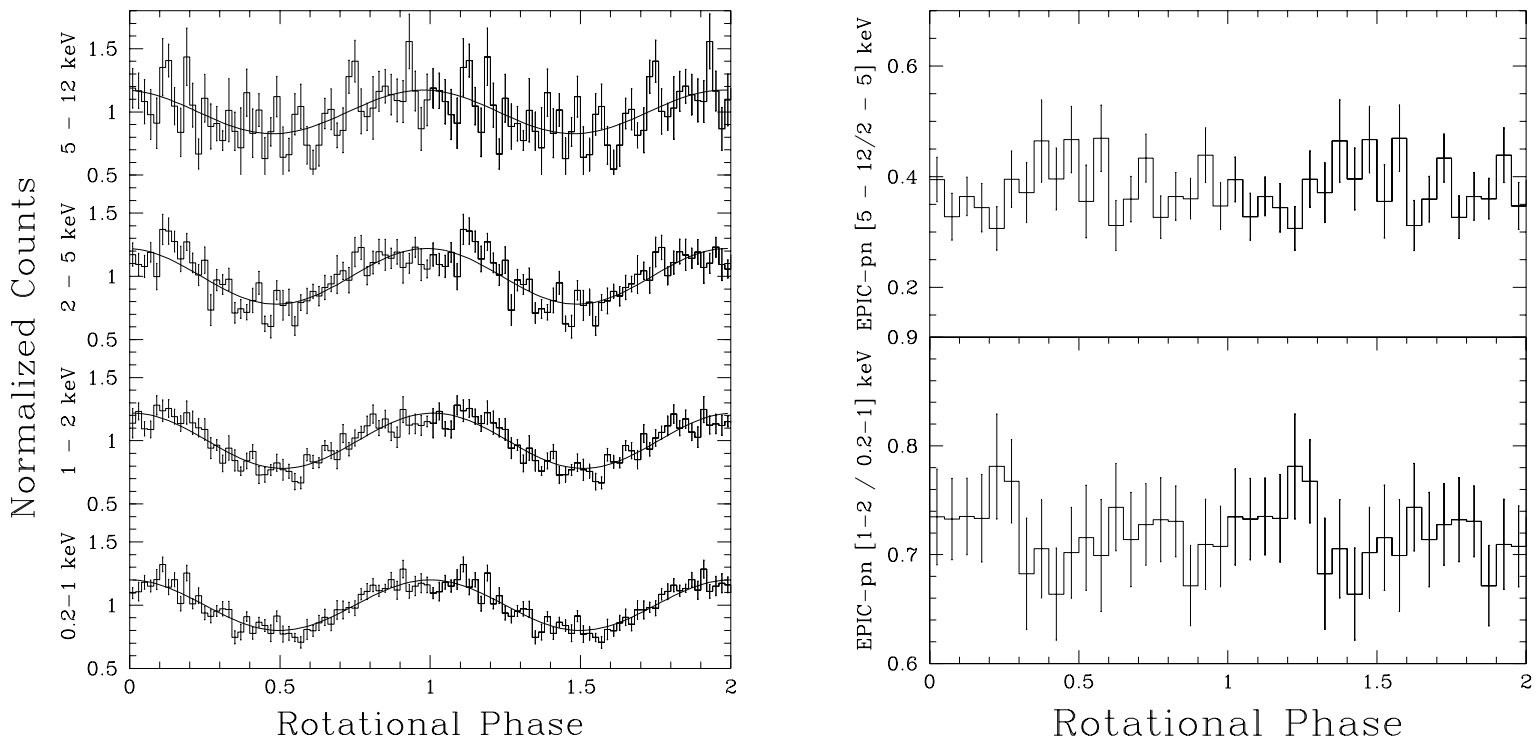

Fig. 3. Left: EPIC-PN folded light curves in selected energy bands at the $515 \mathrm{~s}$ period using the ephemeris quoted in the text together with the best sinusoidal fit. Right: the EPIC hardness ratios show no energy dependence of the pulse.

the PN data for the time of maximum of X-ray spin pulse: $\mathrm{HJD}_{\max }=2452723.28316(4)+0.005956(2) \mathrm{E}$. Here we note that the time of X-ray maximum is consistent with that predicted by the accurate ephemeris by Kemp et al. (2002), which is derived from data taken between 1997 and 2002.

The periodic variability was also explored at different energies in the EPIC range. Light curves were also extracted with the same $50 \mathrm{~s}$ binning time in the four ranges: $0.2-1 \mathrm{keV}$, $1-2 \mathrm{keV}, 2-5 \mathrm{keV}$ and $5-12 \mathrm{keV}$ and Fourier analysed as shown in Fig. 2. The spin peak is strong at all energies except for the harder range where it is detected at $2.8 \sigma$. The first and second harmonics are detected in the $1-5 \mathrm{keV}$ range. Pulse folded light curves were constructed in the above ranges using the ephemeris given above. These are shown in Fig. 3, together with the hardness ratios defined as the ratio of countrates in [5-12 keV] and [2-5 keV] ranges and in [1-2 keV] and $[0.2-1 \mathrm{keV}]$ ranges. The light curves are quasi-sinusoidal and display a broad maximum with an additional peak which is best visible in the $2-5 \mathrm{keV}$ band. The modulation has fractional amplitudes: $40 \pm 2 \%$ in $0.2-1 \mathrm{keV}, 43 \pm 5 \%$ in $1-2 \mathrm{keV}, 44 \pm$ $7 \%$ in $2-5 \mathrm{keV}$, and $34 \pm 15 \%$ in $5-12 \mathrm{keV}$ band. Hence, within the errors the pulsation has the same amplitudes at all energies. The constancy of hardness ratios with spin phase further confirms the lack of energy dependence of pulse amplitudes.

\subsection{The RXTE light curve}

Background subtracted light curves were extracted in channels 5-13 (2-6 keV) and 14-23 (6-10 keV). A Fourier analysis reveals power at the known pulse frequency, but the peaks are not statistically significant. Hence, while a detection of the spin period from the RXTE data alone cannot be claimed, the RXTE data are consistent with the known pulse period. To fold light curves at the spin period, we use the Kemp et al. (2002) ephemeris since our X-ray ephemeris are not accurate enough to extrapolate the XMM-Newton to RXTE observations.

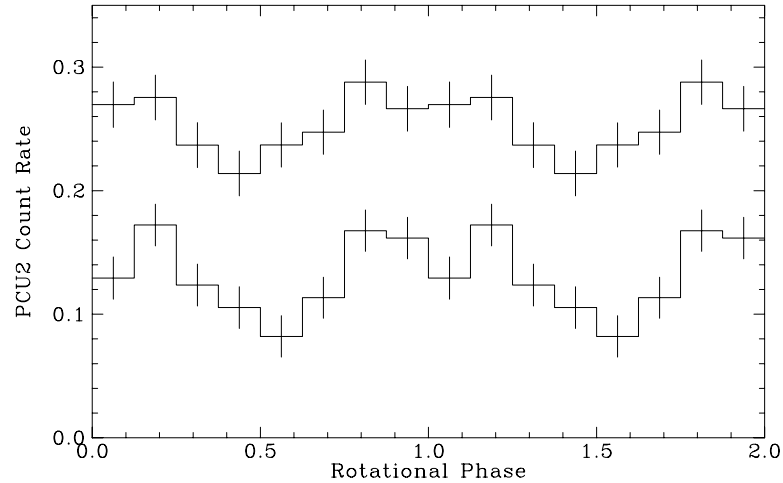

Fig. 4. The RXTE spin folded light curves in the $2-6 \mathrm{keV}$ (higher curve) and 6-10 keV (lower curve) bands shown without offset, using Kemp et al. (2002) ephemeris as discussed in the text.

The folded light curves in these two bands are shown in Fig. 4. The modulation is present in both bands and very similar to that observed with great details in the EPIC data. However, given that the source is close to the systematic limit of RXTE detection, which can affect the average count rate of the source especially in the harder band, we are unable to assess on the relative pulse amplitude in the two bands. We then limit ourselves to confirm the presence of X-ray pulse and that the maximum of the modulation is in phase with the optical spin ephemeris previously determined (see also Sect. 4.2).

\section{Optical and UV timing analysis}

\subsection{The Optical Monitor data}

The XMM-Newton OM B and UVW2 filter $10 \mathrm{~s}$ light curves were inspected for periodic signals. A discrete Fourier analysis (DFT) is shown in the left panel of Fig. 5, where the $515 \mathrm{~s}$ pulse is clearly evident in the B band but not in the UV range. The CLEAN algorithm has also been applied thus removing most 

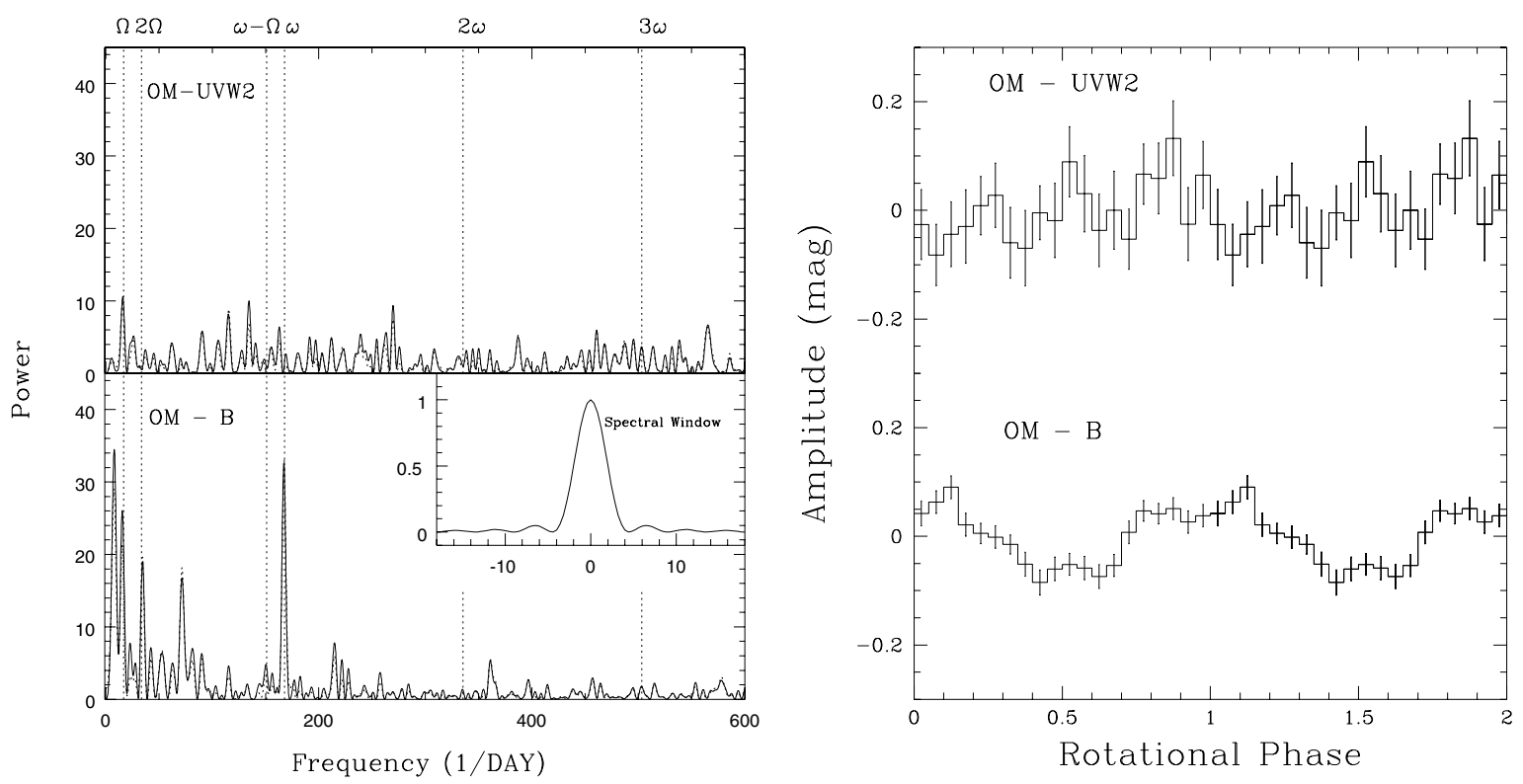

Fig. 5. Left: DFTs (solid line) of $B$ and $U V W 2$ time series, together with that using the CLEAN algorithm (dashed line). Right: folded light curves at the $515 \mathrm{~s}$ spin period using the X-ray ephemeris quoted in the text. Ordinates are modulation amplitudes.
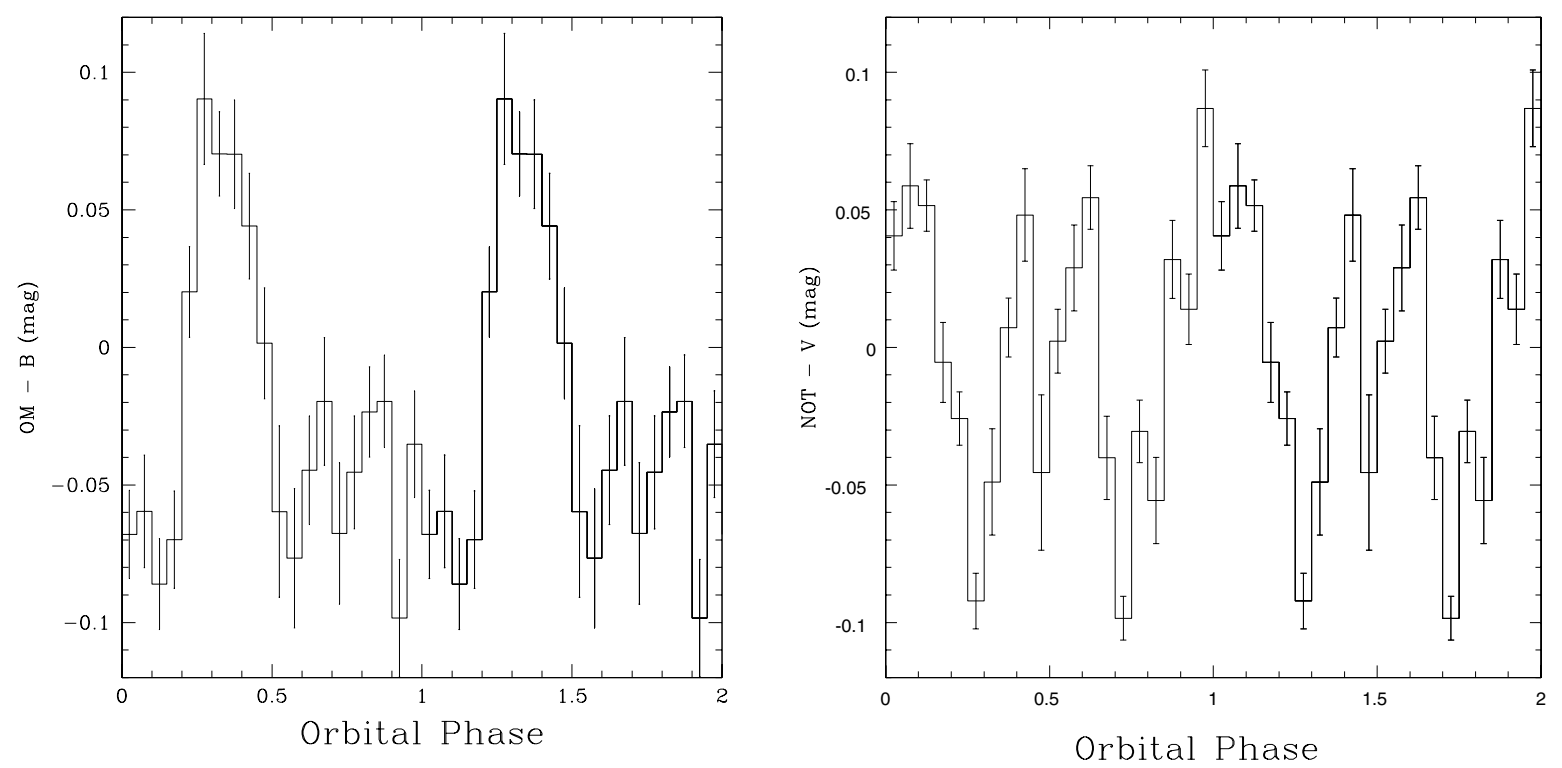

Fig. 6. The March 2003 OM B band (left) and the December 2002 NOT V band (right) folded light curves at the orbital 85.98 min period after pre-whitening the data from the high frequency spin pulse. Phase zero has an arbitrary origin. Ordinates are modulation amplitudes.

of the windowing effects of OM data acquisition. No power is detected at the beat $(\omega-\Omega)$ frequency or at the first and second harmonics of the $515 \mathrm{~s}$ signal. Power is present close to the orbital $\left(16.7 \mathrm{day}^{-1}\right)$ frequency in the $B$ and $U V$ bands although in this latter range the signal is very weak. Power is also detected at the first harmonic of the orbital frquency in the $B$ band only. A sinusoidal fit to the $B$ band light curve with two frequencies, accounting for the spin and orbital modulations, gives $\omega=167.06 \pm 0.26 \mathrm{day}^{-1}$ and $\Omega=15.22 \pm$ $0.27 \mathrm{day}^{-1}$. The spin period agrees within $3 \sigma$ with that determined by Kemp et al. (2002), while the orbital period is off by $\sim 5 \sigma$. The $B$ and $U V W 2$ band light curves were then folded using the pulse ephemeris derived from the EPIC data and are shown in the right panel of Fig. 5. The $B$ band pulse fraction is $0.13 \pm 0.01 \mathrm{mag}$ consistent with previous detections and its shape is not sinusoidal with a broad maximum and a dip overimposed on it as well as a structured minimum. In order to inspect the orbital variability, the $B$ band light curve was prewhitened by the $515 \mathrm{~s}$ signal and then folded at the $85.98 \mathrm{~min}$ spectroscopic orbital period determined by Kemp et al. (2002). However, their spectroscopic orbital ephemeris is not accurate enough to be used for comparison between the zero crossing of radial velocities of emission lines and the photometric maximum. In Fig. 6 (left panel), we report the orbital light curve 


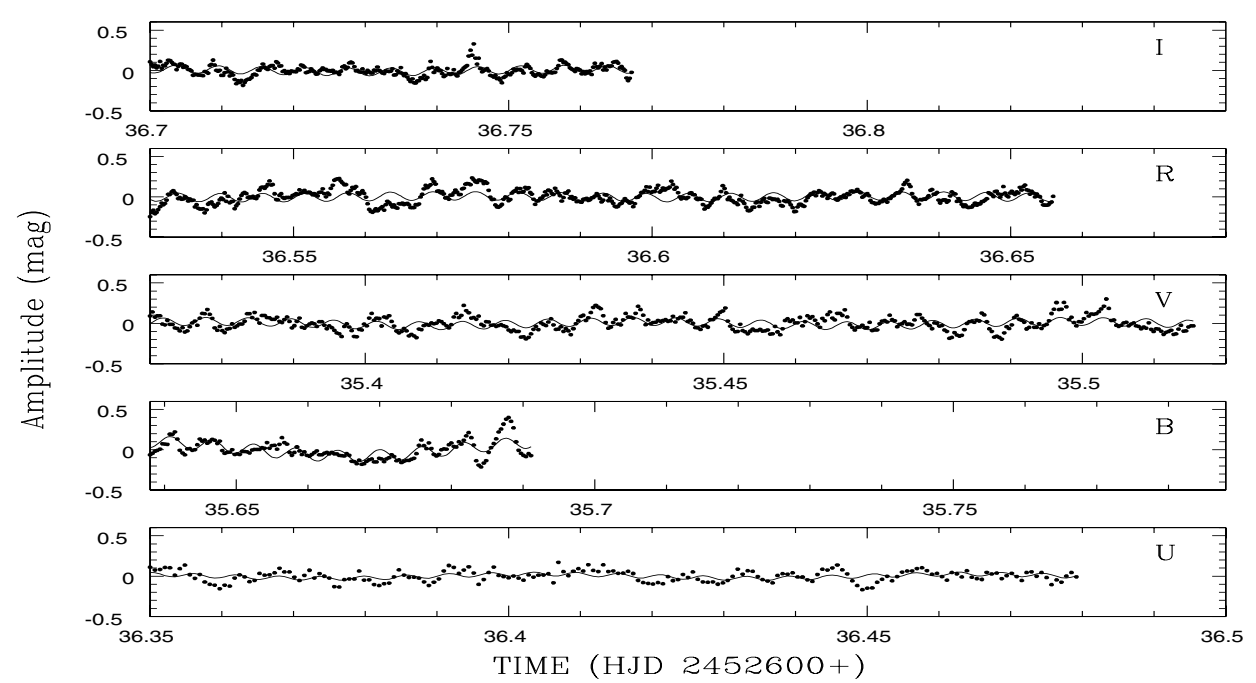

Fig. 7. The UVBRI NOT light curves together with their best fit sinusoidal function with a period of $515 \mathrm{~s}$. For the $B$ band light curve a polynomial function accounting for the low frequency trend has been included. Ordinates are modulation amplitudes.
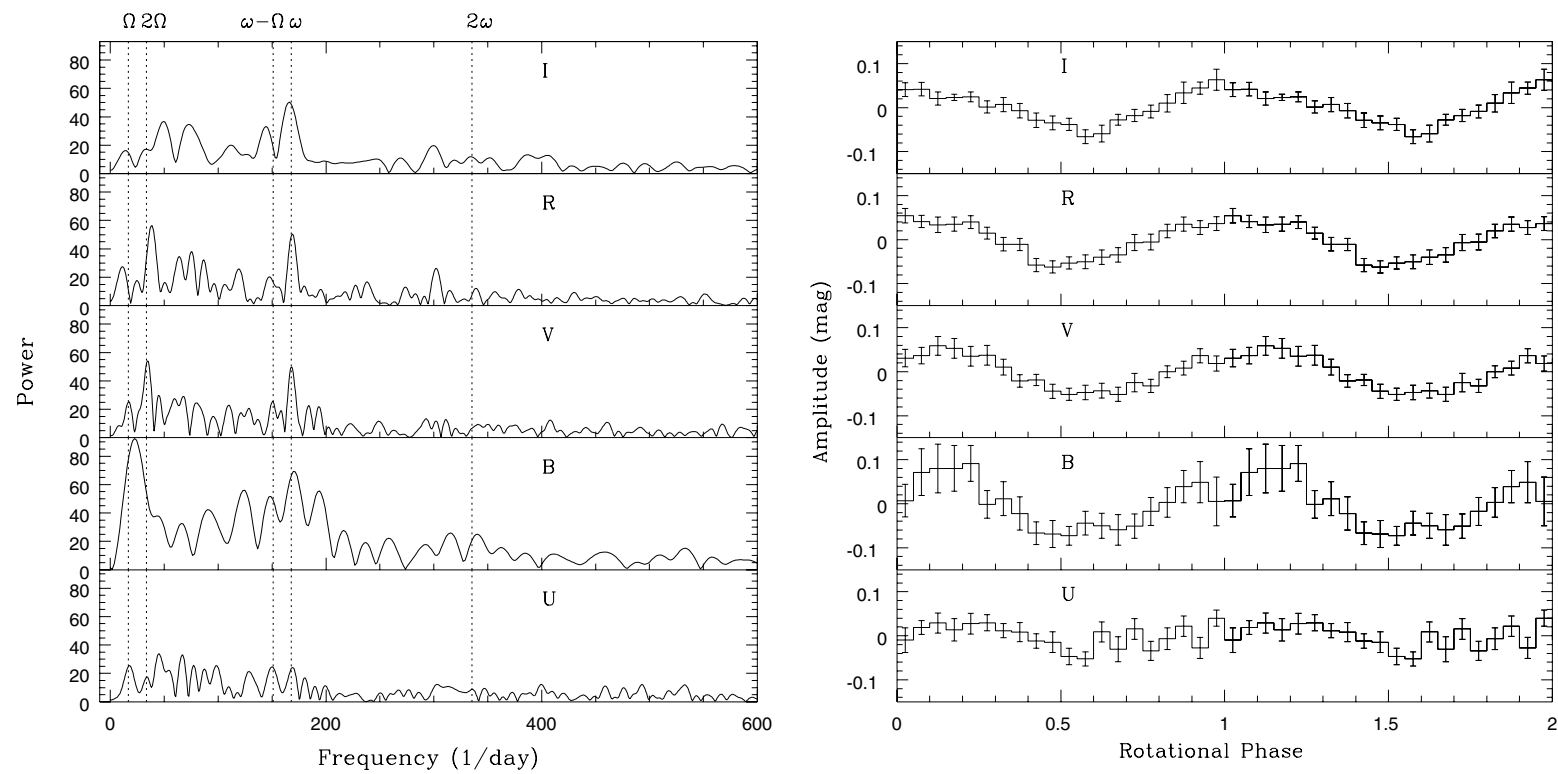

Fig. 8. Left: the DFTs of UBVRI light curves show a that the spin pulse is present in all bands except in the $U$. Right: folded light curves at the 515 s period using Kemp et al. (2002) ephemeris. These confirm the lack of UV pulsation but a strong optical modulation at longer wavelengths. Ordinates are modulation amplitudes.

which shows a strongly peaked modulation with a full amplitude of $0.14 \pm 0.02 \mathrm{mag}$. The strongly non-sinusoidal square shape of the modulation explains the presence of the first harmonic of the orbital frequency. Such variability in quiescence was not reported previously.

\subsection{The UBVRI light curves}

The UBVRI light curves confirm the behaviour observed in the OM data (Fig. 7), the $U$ band being unmodulated. Fourier analysis of the time series is shown in the left panel of Fig. 8, where the $515 \mathrm{~s}$ pulsation is clearly detected longward of the $B$ band. Power at the beat frequency is not statistically significant but we detect power close to the first harmonic of the orbital frequency in the $V$ and $R$ bands, which are the longest runs. Sinusoidal fits to the light curves consisting of one frequency give $\omega(B)=171.36 \pm 1.41 \mathrm{day}^{-1}$ (this does not improve when including a second frequency fixed at the orbital period), $\omega(V)=167.8 \pm 0.4 \mathrm{day}^{-1}, \omega(R)=168.6 \pm 0.4 \mathrm{day}^{-1}$ and $\omega(I)=$ $166.0 \pm 0.9$ day $^{-1}$ (see Fig. 7). These determinations are within errors consistent with that found by Kemp et al. (2002). Given the low accuracy of the frequency and the time of maximum determination, even in the longest run with the $V$ filter, we use the optical ephemeris by Kemp et al. (2002) to construct spin folded light curves. The pulses (right panel of Fig. 8) are strong at longer wavelengths but roughly similar (within errors) in all bands. The full amplitudes are $0.13 \pm 0.01 \mathrm{mag}$ in the $B$, 


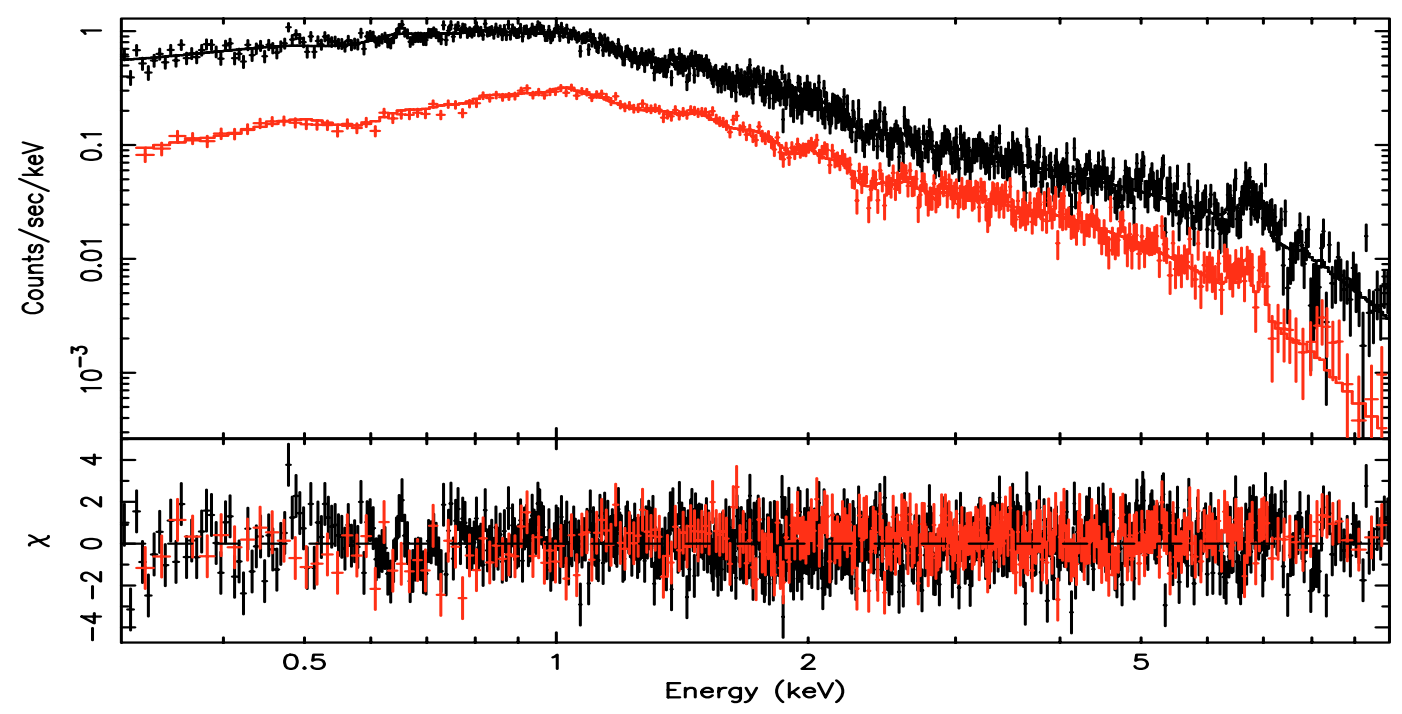

Fig. 9. The EPIC PN (top) and combined MOS (bottom) spectra fitted simultaneously with a multi-temperature model with $k T_{\max }=19 \mathrm{keV}$ and power law index $\alpha=0.72$ absorbed by a column with density $N_{\mathrm{H}}=5.9 \times 10^{20} \mathrm{~cm}^{-2}$ plus a Gaussian centred at $6.4 \mathrm{keV}$ to account for the fluorescent iron line. The bottom panel shows the residuals.

$0.10 \pm 0.01 \mathrm{mag}$ in the $V, 0.10 \pm 0.01 \mathrm{mag}$ in the $R$ and $0.09 \pm$ 0.01 mag in the $I$ band. A comparison with the OM $B$ band light curve shows consistency with a structured maximum and a dip superposed on it. This feature seems to disappear moving towards the red. As found in the simultaneous X-ray and optical observations of March 2003, also during the December 2002 observations, the X-ray and optical pulses are in phase.

We have further inspected the data against orbital variability in the long runs with the $V$ and $R$ filters. While we are unable to determine the orbital frequency in the $R$ band, we find $P_{\text {orb }}=0.059 \pm 0.003$ day in the $V$ filter which is consistent with the previous optical determinations. We then detrended the $V$ filter light curve from the spin modulation and folded the data at the orbital period determined by Kemp et al. (2002). Figure 6, (right panel) shows a structured light curve with a broad primary maximum and a double-peaked second one offsetted by 0.5 in phase. This explains the lack of power at the orbital frequency but at its first harmonic. Given that the $B$ band coverage is less than one orbital period, we have compared the shape of the $V$ band light curve with that observed in March 2003 in the $B$ filter and it appears that the structured minimum in the latter has developed into two separate peaks. The full amplitude of the variability being $\sim 0.15 \mathrm{mag}$.

\section{The X-ray spectra}

The average EPIC PN and combined MOS spectra were analysed with the XSPEC package. Due to calibration accuracy issues, the spectra have been analysed between 0.3 and $10 \mathrm{keV}$. An absorbed simple isothermal optically thin emission model MEKAL plus a Gaussian centred at $6.4 \mathrm{keV}$ does not provide an acceptable fit $\left(\chi_{v}^{2}=20\right)$. The accretion post-shock regions are expected to possess a temperature gradient due to the cooling of the gas approaching the WD surface. We then used a composite model consisting of a multi-temperature optically thin plasma CEMEKL with a power law temperature distribution with index $\alpha$ and maximum temperature $k T_{\max }$ and metal abundance $A_{Z}$ in number with respect to solar, plus a zero-width (unresolved) Gaussian fixed at $6.4 \mathrm{keV}$ and a total absorber with a hydrogen column density $N_{\mathrm{H}}$. This model gives a much better agreement as shown in Fig. 9. The best fit spectral parameters, together with the $90 \%$ confidence level for the interesting parameter, are reported in Table 2 . The hydrogen column density of the absorber is unusually low and consistent with the galactic hydrogen column density in the direction of the source $N_{\mathrm{H}, \mathrm{gal}}=4.3 \times 10^{20} \mathrm{~cm}^{-2}$ (Dickey \& Lockman 1990). Hence, while most IPs suffer from strong and sometimes very complex absorption (see Mukai et al. 1994), it seems that HT Cam does not share this general characteristic. Also, assuming that the maximum temperature represents that of the shock, $\left(k T_{\max }=k T_{\text {shock }}=3 / 8 \mathrm{G} M_{\mathrm{WD}} \mu m_{\mathrm{H}} R_{\mathrm{WD}}^{-1} \mathrm{keV}\right.$, where $\mu$ is the mean molecular weight and $m_{\mathrm{H}}$ the mass of hydrogen), the inferred value is not as high as those determined in other systems of this class (Beardmore et al. 2000), but it is similar to that determined in EX Hya (Fujimoto \& Ishida 1997). The metal abundances are sub-solar, although caution has to be taken since these are relative to Anders \& Grevesse solar abundances, whose iron abundance is higher than others available in XSPEC. The low equivalent width $(E W)$ of the fluorescent iron line indicates that reflection is not important in this source and justifies the neglection of this component in the spectral fits.

The EPIC PN and combined MOS spectra were also extracted around pulse maximum $(\phi=0.8-1.2)$ and minimum $(\phi=0.4-0.6)$ and fitted with the same model, keeping fixed the metal abundance to the value found for the average spectrum. To fit the spectrum at minimum also the power law index has been kept fixed to the average value. Leaving free this parameter the temperature slope is less steep $\left(\alpha=0.51_{-0.13}^{+0.14}\right)$, but the shock temperature is badly constrained $\left(k T_{\max }=30_{-10}^{+20} \mathrm{keV}\right.$, $\left.\chi_{v}^{2}=0.80\right)$. As expected from the light curves and hardness ratios at different energies, the only parameter which changes significantly is the normalization, by a factor of $40 \%$, 
Table 2. Spectral parameters as derived from fitting simultaneously the EPIC PN and MOS phase-averaged spectra, those at pulse maximum and minimum as well as from the fits to the RGS1 and RGS2 averaged spectra. Quoted errors refer to 90\% confidence level for the parameter of interest.

\begin{tabular}{|c|c|c|c|c|c|}
\hline & \multicolumn{3}{|c|}{ EPIC } & \multicolumn{2}{|c|}{ RGS } \\
\hline & Average & Pulse maximum & Pulse minimum & Average & Average \\
\hline$F_{2-10}^{a}$ & $3.36 \pm 0.02$ & $3.84 \pm 0.05$ & $2.65 \pm 0.06$ & & \\
\hline$N_{\mathrm{H}}^{b}$ & $5.9_{-0.4}^{+0.7}$ & $5.2 \pm 0.6$ & $6.7_{-1.0}^{+0.9}$ & 5.9 (fixed) & $11.0_{-2.0}^{+3.0}$ \\
\hline$k T_{\max }[\mathrm{keV}]$ & $19 \pm 3$ & $19_{-3}^{+5}$ & $18_{-2}^{+3}$ & 19 (fixed) & 19 (fixed) \\
\hline$\alpha$ & $0.72_{-0.08}^{+0.10}$ & $0.74 \pm 0.1$ & 0.72 (fixed) & 0.72 (fixed) & 0.72 (fixed) \\
\hline$A_{\mathrm{Z}}^{c}$ & $0.59 \pm 0.10$ & 0.59 (fixed) & 0.59 (fixed) & 0.59 (fixed) & 0.59 (fixed) \\
\hline$C_{\text {nor. }}^{d}\left[10^{-3}\right]$ & $5.1_{-0.3}^{+0.4}$ & $6.0_{-0.7}^{+0.6}$ & $3.9 \pm 0.1$ & $3.9 \pm 0.2$ & $4.6_{-0.5}^{+0.4}$ \\
\hline$E W^{e}$ & $50_{-40}^{+34}$ & $27(<80)$ & $100(<150)$ & & \\
\hline$\chi_{v}^{2}\left(\chi^{2} /\right.$ d.o.f. $)$ & $1.02(1082 / 1058)$ & $0.91(561 / 615)$ & $0.81(279 / 344)$ & $0.96(200 / 209)$ & $0.92(191 / 208)$ \\
\hline
\end{tabular}

${ }^{a}$ Flux in units of $10^{-12} \mathrm{erg} \mathrm{cm} \mathrm{cm}^{-2} \mathrm{~s}^{-1}$ in the $2-10 \mathrm{keV}$ band.

${ }^{b}$ Column density of the absorber in units of $10^{20} \mathrm{~cm}^{-2}$.

${ }^{c}$ Metal abundance in units of the cosmic value (Anders \& Grevesse 1989).

${ }^{d}$ Normalization constant of CEMEKL model.

${ }^{e}$ Equivalent width of the $6.4 \mathrm{keV}$ fluorescent iron line in units of $\mathrm{eV}$.

Table 3. Line parameters as measured in the RGS spectra of HT Cam.

\begin{tabular}{cccc}
\hline \hline Line & $\begin{array}{c}E_{\mathrm{C}} \\
(\mathrm{keV})\end{array}$ & $\begin{array}{c}\sigma \\
(\mathrm{eV})\end{array}$ & $\begin{array}{c}\text { Flux } \\
\mathrm{ph} \mathrm{cm}^{-2} \mathrm{~s}^{-1}\end{array}$ \\
\hline Blend & $0.8213_{-0.0044}^{+0.0046}$ & $6.2_{-3.4}^{+3.8}$ & $2.0 \pm 0.9 \times 10^{-5}$ \\
O VIII & $0.6536_{-0.0009}^{+0.0011}$ & $2.4_{-1.3}^{+1.4}$ & $3.3 \pm 0.9 \times 10^{-5}$ \\
O VII & $0.5723_{-0.0030}^{+0.0031}$ & $2.8(<5.8)$ & $2.1_{-1.3}^{+1.5} \times 10^{-5}$ \\
\hline
\end{tabular}

the absorption remaining constant within errors. A further check has been done by only varying the absorption and keeping fixed the normalization when fitting the pulse minimum spectrum. While absorption increases up to $1.4 \times 10^{21} \mathrm{~cm}^{-2}$ (or $1.0 \times 10^{21} \mathrm{~cm}^{-2}$ ) when fixing the normalization at the pulse maximum value (or at the average spectrum value), the fit quality is much worse, $\chi_{v}^{2}=2.55$ (or $\chi_{v}^{2}=1.53$ ). Furthermore, the $E W$ of fluorescent iron line is not constrained although there is indication that it is stronger at pulse minimum, likely due to the changes in the continuum level. The best spectral fit parameters at these phases are also reported in Table 2.

In the RGS spectra, we detect the O VIII (19.1 $\mathrm{A}) \mathrm{Ly}_{\alpha}$ line with high degree of confidence, including the line width $(\sigma \sim$ $\left.1100 \pm 600 \mathrm{~km} \mathrm{~s}^{-1}\right)$. The O VII $(21.9 \AA)$ He-like triplet is also detected. The oxygen region of the RGS spectra are shown in Fig. 10. The detected O VII line is likely to be a blend of the resonant (r) and intercombination (i) lines, whereas there is no evidence for the forbidden (f) line. We also detect a feature around $15.1 \AA$, which is likely to be a blend including the Fe XVII 15.01 $\AA$ line. There is an excess of counts around $17 \AA$ that is consistent with the Fe XVII lines expected there, given the presence of the $15.1 \AA$ blend. However, the $17 \AA$ feature is too weak to provide reliable numbers. The measured line parameters are reported in Table 3.

The same multi-temperature model used for EPIC spectral fits provides an adequate description of the RGS spectra.

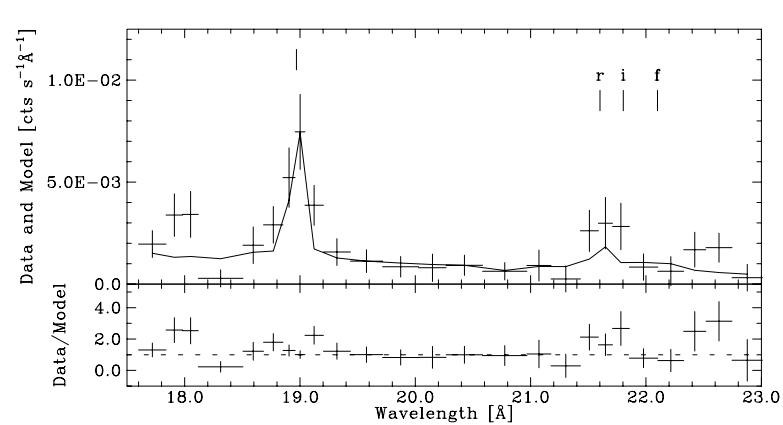

Fig. 10. The region including the oxygen lines of the RGS spectra, together with the EPIC best fit model. The rest wavelengths of the lines are also marked. The positions for the forbidden (f), intercombination (i) and resonance (r) lines are displayed.

We obtain $\chi_{v}^{2}=0.97$ by just adjusting the normalization, with all other parameters $\left(T_{\max }, \alpha, A_{Z}\right.$ and $\left.N_{\mathrm{H}}\right)$ fixed to the EPIC spectral fit values). The resulting normalization for the RGS spectra is somewhat smaller than the best-fit EPIC value. We obtain a better fit $\left(\chi_{v}^{2}=0.92\right)$ allowing absorption to vary; the best-fit $N_{\mathrm{H}}$ value is higher $\left(1.1 \times 10^{21} \mathrm{~cm}^{-2}\right)$ than in the EPIC spectral fit, while the normalization is in better agreement. Because of the limited statistical quality, potential systematic errors in the background subtraction, and/or spectral complexity (HT Cam may have low level partial covering absorber, as is seen in EX Hya, Cordova et al. 1985), we do not consider these discrepancies to be serious. Instead, we take the success of the CEMEKL fit as confirming the multi-temperature nature of the X-ray emitter in HT Cam.

\section{Discussion}

Our X-ray observations of HT Cam reveal a strong (40\%) pulsation at the $515 \mathrm{~s}$ optical period, which unambiguously assigns this CV to the IP class of magnetic systems. It is then the third 
confirmed IP, along with V 1025 Cen and EX Hya, below the $2-3 \mathrm{~h}$ orbital period gap.

\subsection{The pulsator in HTCam}

The X-ray power spectrum does not reveal further periodic signals, such as sidebands, which indicates that the X-ray period is indeed the rotational period of the WD and that accretion occurs via a disc (Norton et al. 1996). The X-ray pulses are quasi-sinusoidal as in most IPs, but different from the majority of these systems they are not energy dependent. In the classical accretion scenario, the accreting material falls onto the WD poles from the inner edge of the truncated disc at the magnetospheric radius and flows along the magnetic field lines in an arc-shaped curtain (Rosen et al. 1988). In this model, the optical depth of the infall gas in the curtain is larger along the field lines rather than perpendicularly and hence photoelectric absorption is larger when the curtain points towards the observer. As for the short orbital period IPs, EX Hya (Rosen et al. 1988) and V 1025 Cen (Hellier et al. 1998), the mechanisms causing the pulsations are a combination of photo-electric absorption in the curtain and occultation. In HT Cam, instead there is no evidence for phase-dependent absorption. This suggests that the rotational pulses are only due to aspect changes of the X-ray emitting region, i.e. occultation of this region as the WD rotates. The changes by $40 \%$ of the normalization implies that the emission measure of the X-ray emitting source changes from $4.7 \times 10^{53} d_{100}^{2} \mathrm{~cm}^{-3}$ at pulse minimum to $7.2 \times 10^{53} d_{100}^{2} \mathrm{~cm}^{-3}$ at pulse maximum, where $d_{100}$ is the distance in units of $100 \mathrm{pc}$. Hence, although we cannot exclude that a secondary pole is also visible, it is reasonable to assume that the main accreting pole does not completely disappear behind the WD limb.

The optical pulses are in phase with the X-ray modulation, similarly to most IPs. This implies that the optical pulsation originates in the magnetically confined accretion flow. The pulse amplitudes in our colour photometry are quite similar in all bands $(\sim 10 \%)$ except in the UV where both far-UV and near-UV ranges are unmodulated. This is an unusual behaviour, since the UV flux is known to be modulated at the rotational period of the WD in other IPs, like AE Aqr (Eracleous \& Horne 1994), YY Dra (Haswell et al. 1997), FO Aqr (de Martino et al. 1999), EX Hya (Eisenbart et al. 2002). In these systems the UV pulses originate in the heated polar region of the WD atmosphere. Hence, the lack of UV modulation in HT Cam suggests that heating of the WD poles is negligible. Also, it is very unlikely that the reprocessing of hard X-rays peaks out of the UV window, since a soft X-ray component is not observed in the X-rays. Furthermore, the optical pulsations in IPs are typically colour dependent (Welsh \& Martell 1996; de Martino et al. 1999; Eisenbart et al. 2002) and the pulse spectrum is generally described by a blackbody function. In HT Cam the lack of colour dependence, together with the absence of UV pulses is difficult to reconcile with optically thick emission. An alternative possibility is that the optical spin pulses are due to aspect angle changes of a region in the accretion flow which is optically thin but cool enough to not affect the Balmer jump. The low hydrogen column density found in the X-rays might corroborate this idea, but further photometry extending to the IR and phase-resolved optical spectrophotometry are required to clarify this puzzle.

We also detect an orbital strongly peaked modulation in the $B$ band photometry from the OM instrument (March 2003) with full amplitude of $0.14 \mathrm{mag}$. This is also detected in the December 2002 NOT run but the modulation appears to have changed into a complex double structured shape. This variability during quiescence was not reported previously, but only when HT Cam was in outburst. We are confident that HT Cam was in quiescence during both optical observations in December 2002 and March 2003. It might be possible that the orbital modulation was over-looked in previous optical photometric data sets.

\subsection{The post-shock region}

Both EPIC and RGS spectra reveal that the X-ray post-shock emitting region has a multi-temperature structure. This is expected by the standard accretion shock model, where matter falls onto the WD almost radially at free-fall velocities thus producing a stand-off shock below which gas cools via thermal bremsstrahlung as it descends and settles on the WD surface (Aizu 1973). From the ratio of oxygen lines, which particularly map the low temperatures, and the maximum temperature determined from the spectral fits, the plasma temperature extends from $k T_{\text {min }}=0.3_{-0.1}^{+0.2} \mathrm{keV}$ to $k T_{\text {max }} \sim 19 \pm 3 \mathrm{keV}$. This range of temperature is, within errors, close to that found in EX Hya (Fujimoto \& Ishida 1997). To infer the post-shock densities, the standard diagnostic of high-temperature plamas is the ratio of forbidden to intercombination lines of He-like ions $(R=f / i)$. This ratio decreases with increasing densities. In HT Cam the forbidden line of O VII is not detected, suggesting a high density limit (Porquet \& Dubau 2000). However, the quality of the RGS spectrum can provide only a $1 \sigma$ lower limit on the electron density: $n_{\mathrm{e}}>5 \times 10^{12} \mathrm{~cm}^{-3}$. We also note that the emission measure derived above is remarkably in agreement with that expected from post-shock regions. For a typical polar cap $\sim 10^{16} \mathrm{~cm}^{2}$ and shock height of $10^{8} \mathrm{~cm}$, the emission measure gives a gas density of $n \sim 8 \times 10^{14} d_{100} \mathrm{~cm}^{-3}$, which is consistent with the high density limit estimated from the RGS data. However, as stressed by Porquet et al. (2001) and Mauche et al. (2001) a strong UV radiation field, as present in CVs, can mimic a high density regime, implying that the upper level of the forbidden line can be significantly depopulated via photo-excitation to the upper levels of intercombination lines. As for the O VII line, a blackbody radiation with temperature of $>30000 \mathrm{~K}$ can produce a strong decrease in the intensity of the forbidden line and an increase of the intercombination line.

Furthermore, expected typical post-shock velocities in magnetic CVs are $v \leq v_{\text {shock }}=v_{\text {ff }} / 4 \sim 900 \mathrm{~km} \mathrm{~s}^{-1}$, where $v_{\mathrm{ff}}=\left(2 \mathrm{G} M_{\mathrm{WD}} / R\right)^{1 / 2}$ is the free-fall veolcity in the preshock flow. The optical spectrum of HTCam (Tovmassian et al. 1998; Kemp et al. 2002) shows broad emission lines (FWHM 3000-4000 km-1), while the width of the X-ray oxygen lines, though not well constrained, are narrower 
$\left(\sigma \sim 1000 \pm 600 \mathrm{~km} \mathrm{~s}^{-1}\right)$. Hence, the X-ray lines are broadly compatible with post-shock velocities.

Using the maximum temperature derived from the X-ray spectral fits $(19 \pm 3 \mathrm{keV})$ as the shock temperature and adopting the WD mass-radius relation of Nauenberg (1972), we derive a WD mass $M_{\mathrm{WD}}=0.55 \pm 0.09 M_{\odot}$. This value falls in the low mass range of $\mathrm{CV}$ masses and it is similar to that determined for EX Hya (Fujimoto \& Ishida 1997; Cropper et al. 1998).

The weak fluorescent "neutral" $\mathrm{Fe}$ line at $6.4 \mathrm{keV}$ indicates that reflection from cold material is not important in HT Cam. The origin of the fluorescent line in magnetic CVs is likely reflection of hard X-rays at the WD surface (Beardmore et al. 2000; Matt et al. 2000; de Martino et al. 2001, 2004), although the pre-shock material can be also an additional source (Ezuka \& Ishida 1999). However, the $90 \%$ confidence level lower limit to the $E W$ of the $6.4 \mathrm{keV}$ line is too large to be produced by a pre-shock gas with hydrogen column density $<10^{21} \mathrm{~cm}^{-2}$, implying that this line originates by reflection at the WD surface.

\subsection{HTCam: A low mass transfer rate system}

The hydrogen column density of the absorbing material above the shock is found to be lower by two orders of magnitude than typical values of IPs (Ezuka \& Ishida 1999). Here we note that also the other two IPs below the gap, EX Hya (Mukai et al. 2003) and V1025 Cen (Hellier et al. 1998) are characterized by low absorbing columns, although these two possess phasedependent complex partial absorbing material. Hence, the lack of a high density absorber above the source suggests that the mass accretion rate is low. The large $E W$ s of optical Balmer lines and the weak He II emission feature (Tovmassian et al. 1998) are now understandable in this context. We use the unabsorbed bolometric flux derived from spectral fits which gives: $L_{\text {bol }}=9.0 \times 10^{30} d_{100}^{2} \mathrm{erg} \mathrm{s}^{-1}$. Equating this to the accretion luminosity $L_{\text {accr. }}=\mathrm{G} \dot{M}_{\odot} M_{\mathrm{WD}} / R_{\mathrm{WD}}$ and assuming the derived WD mass $M_{\mathrm{WD}}=0.55 M_{\odot}$, we derive $\dot{M}=2.4 \times$ $10^{-11} d_{100}^{2} M_{\odot} \mathrm{yr}^{-1}$. The predicted accretion rate for a system with $P_{\text {orb }}=86 \mathrm{~min}$ is $\dot{M} \sim 2 \times 10^{-11} M_{\odot} \mathrm{yr}^{-1}$. Hence, unless the distance is as large as that estimated by Tovmassian et al. (1998) $(d=400 \mathrm{pc})$, the accretion rate is consistent with that predicted by gravitational radiation and similar to that recently found in EX Hya (Beuermann et al. 2003). We note that, Tovmassian et al. (1998) estimate the distance for HT Cam from $K$-band flux assuming a M $8 \mathrm{~V}$ secondary star with radius similar to normal field dwarf stars. Probably this is unlikely (see also below), but also because the mass accretion rate would be too high for transfer rates expected for short orbital period systems (Patterson 1998).

Hence, HTCam shares the low accretion rates with the other two IPs below the period gap, EX Hya and V1025 Cen, but differently from these weakly de-synchronized systems, the spin-to-orbital period ratio is close to 0.1 . This value is the one expected for systems spinning at equilibrium by accreting the specific angular momentum of the secondary star (King \& Lasota 1991). Indeed, Kemp et al. (2002) find that the spin period derivative in HT Cam is small. Thus HT Cam is located at the border line in the spin-orbital period plane above which accretion does not occur predominantly via a disc. However, for the three short-period systems indications of disc accretion are found. Assuming spin equilibrium and disc accretion, a value of the magnetic moment $\mu$ of the WD can be obtained: $\omega_{\mathrm{s}}=0.041 P_{1000}^{-1} \dot{M}_{17}^{-3 / 7} M_{\mathrm{WD}}^{-5 / 7} \mu_{32}^{6 / 7}$ where $\omega_{\mathrm{s}}=0.6$ (Patterson $1994)$ is the fastness parameter which is valid for rapid rotators like HT Cam, $P_{1000}$ is the spin period in units of $1000 \mathrm{~s}, \dot{M}_{17}$ is the accretion rate in units of $10^{17} \mathrm{~g} \mathrm{~s}^{-1}$ and $\mu_{32}$ is the WD magnetic moment in units of $10^{32} \mathrm{G} \mathrm{cm}^{3}$. Using the values inferred for the WD mass and the mass accretion rate, the magnetic moment can be estimated: $\mu_{32}=0.79 d_{100}$. Hence, the fast rotator and low accretor in HT Cam appears to possess a weak magnetic field comparable to YY Dra, which is also rotating and accreting at a similar rate, although it is likely far from equilibrium (Patterson 1994). Hence, there is a large difference with EX Hya and V1025 Cen as they possess slowly rotating WDs and stronger magnetic fields. We here note that Norton et al. (2004) derive $\mu_{32}=2.7$ for HT Cam from their model of magnetic accretion, but they assume a mass ratio $q=M_{\mathrm{sec}} / M_{\mathrm{WD}}=$ 0.5 and a WD mass of $0.7 M_{\odot}$. Since the magnetic moment controls the spin rate (Patterson 1994), it might be reasonable to suppose that HT Cam will spin up and spin-down around equilibrium value and, given its short orbital period, probably it will never synchronize.

With the derived WD mass it is possible to obtain information on the mass of the donor star. From the orbital period and the WD orbital velocity $V_{\mathrm{WD}}$ the mass function can be computed as: $f(m)=\left(M_{\mathrm{sec}} \sin i\right)^{3} /\left(M_{\mathrm{WD}}+M_{\mathrm{sec}}\right)^{2}=$ $\left(V_{\mathrm{WD}}\right)^{3} P_{\mathrm{orb}} / 2 \pi \mathrm{G}$, where $M_{\mathrm{sec}}$ is the mass of the secondary star and $i$ the inclination of the orbit to the line of sight. For HT Cam, assuming that the emission line velocities $K=(69 \pm$ 4) $\mathrm{km} \mathrm{s}^{-1}$ (Kemp et al. 2002) trace the WD orbital motion (this value is also similar to that in EX Hya, Eisenbart 2002), the mass function is found to be: $f(m)=(2.03 \pm 0.35) \times$ $10^{-3} M_{\odot}$. Assuming our derived WD mass: $M_{\mathrm{sec}}=0.09 M_{\odot}$ for $i=90^{\circ}, M_{\mathrm{sec}}=0.14$ for $i=45^{\circ}$ and $0.21 M_{\odot}$ for $i=30^{\circ}$. Since no X-ray eclipses are observed, $i<75^{\circ}$. Also, from the presence of double-peaked emission lines in the optical spectrum with half-peak separation of $250 \mathrm{~km} \mathrm{~s}^{-1}$ (Kemp et al. 2002), it is possible to further constrain the system inclination. Since these originate in the accretion disc (see Warner 1995), the velocity separation of the peaks is: $V_{\text {peak }}=1154\left(M_{\mathrm{WD}} / M_{\odot}\right)^{1 / 2}\left(f_{\text {Lobe }} R_{\text {Lobe }} / 10^{10} \mathrm{~cm}\right)^{-1 / 2} \sin i$, where $f_{\text {Lobe }}$ is the filling fraction of the primary star Lobe, usually taken as $90 \%$. Adopting $M_{\mathrm{WD}}=0.55 M_{\odot}$ and $M_{\mathrm{sec}}=$ $0.1-0.3 M_{\odot}$, then $R_{\text {Lobe }}=1.82-2.05 \times 10^{10} \mathrm{~cm}$ and hence $V_{\text {peak }}=630-660 \sin i \mathrm{~km} \mathrm{~s}^{-1}$. From the observed value of peak separation, this yelds to $i \sim 25^{\circ}$. For comparison, EX Hya $\left(i=78^{\circ}\right.$, Hellier et al. 1987) has peak separations of $1300 \mathrm{~km} \mathrm{~s}^{-1}$. Hence the secondary mass seems to be further restricted towards high values. For an orbit with $P_{\text {orb }}=86 \mathrm{~min}$, the assumption of a Roche lobe filling companion (Paczynski 1971), leads to a further mass-radius constrain: $R_{\mathrm{L}}=$ $0.0152\left(M_{\mathrm{sec}} / M_{\odot}\right)^{1 / 3}\left(P_{\mathrm{orb}} / 1 \mathrm{~min}\right)^{2 / 3} R_{\odot}$. A comparison with the mass-radius relation for main-sequenc stars (Baraffe et al. 1998 ) indicates that only a very low mass companion $M_{\mathrm{sec}} \leq$ $0.13 M_{\odot}$ will fill its lobe, but for larger values as it seems to be suggested by the very low inclination, a main-sequence 
star will overfill its lobe. In this latter case the companion in HT Cam is significantly overmassive for its size or alternatively its radius is much smaller than that of a main-sequence star of comparable mass. Although the nature of the companion star is beyond the scope of the present work, we note here that HT Cam shows intriguing characteristics which new optical spectrophotometry should address.

\section{Conclusions}

We have presented the first X-ray pointed observations of HT Cam with XMM-Newton and RXTE satellites together with simultaneous optical/UV photometry which firmly confirm that this $\mathrm{CV}$ is an IP member. For its short $86 \mathrm{~min}$ orbital period it is then the third IP below the $2-3 \mathrm{~h}$ period gap. Here we summarize the main results:

- The X-ray emission is dominated by the 515 s optical modulation with no sign of signals at sideband frequencies. This indicates that the X-ray period is the spin period of the WD and that HT Cam mostly accretes via a disc.

- The X-ray pulse is not energy dependent indicating that the mechanism responsible for spin pulsation is occultation of the emitting region while the WD rotates.

- The X-ray spectra show that the post-shock region is multitemperature with a maximum temperature of $\sim 20 \mathrm{keV}$ and that the photo-electric absorption above the shock is very low and constant throughout the spin cycle.

- Oxygen lines detected in the low-energy portion of the $\mathrm{X}$-ray spectrum indicate that the post-shock region is in the high density limit $\left(n_{\mathrm{e}}>5 \times 10^{12} \mathrm{~cm}^{-3}\right)$, athough we cannot exclude that additional/alternative contribution from a strong UV emission is responsible for the lack of the forbidden line.

- From the shock temperature, we estimate the WD mass: $\sim 0.6 M_{\odot}$, which falls into the low value range of WD masses in CVs.

- The optical pulsation shows similar fractional amplitudes longward of the blue, but surprisingly the UV flux is not modulated, indicating that the X-ray heating of the WD poles is not important in this system.

- The mass accretion rate is low: $2.4 \times 10^{-11} d_{100}^{2} M_{\odot} \mathrm{yr}^{-1}$. For $d=100 \mathrm{pc}$ this is close to the expected value for mass transfer rates driven by gravitational radiation.

- The ratio of spin-orbit periods is close to the value predicted for a WD spinning at its equilibrium value and we estimate a magnetic moment: $\log \mu=31.9+\log d_{100} \mathrm{G} \mathrm{cm}^{3}$.

Acknowledgements. Part of this work was supported by the Italian Minister of Research (MURST).The authors wish to thank the XMM-Newton staff at VILSPA and in particular Drs. N. Schartel, P. Rodriguez-Pascual and M. Santos-Leo for their useful suggestions on data analysis. Some of the data presented here have been taken using ALFOSC, which is owned by the Instituto de Astrofisica de Andalucia (IAA) and operated at the Nordic Optical Telescope under agreement between IAA and the NBIfAFG of the Astronomical Observatory of Copenhagen.

\section{References}

Aizu, K. 1973, Prog. Theor. Phys., 49, 1184

Anders, E., \& Grevesse, N. 1989, GeCoA, 53, 197

Baraffe, I., Chabrier, G., Allard, F., \& Hauschildt, P. H. 1998, A\&A, 337, 403

Beardmore, A. P., Osborne, J. P., \& Hellier, C. 2000, MNRAS, 315, 307

Beuermann, K., Harrison, Th. E., Mc Arthur, B. E., et al. 2003, A\&A, 412,821

Cordova, F., Mason, K. O., \& Kahn, S. M. 1985, MNRAS, 212, 447

Cropper, M., Ramsay, G., \& Wu, K. 1998, MNRAS, 293, 222

de Martino, D., Silvotti, R., Buckley, D. A. H., et al. 1999, A\&A, 350, 517

de Martino, D., Matt, G., Mukai, K., et al. 2001, A\&A, 377, 499

de Martino, D., Matt, G., Belloni, T., et al. 2004, A\&A, 415, 1009

den Herder, J. W., Brinkman, A. C., Kahn, S. M., et al. 2001, A\&A, 365, L7

Dickey, J. M., \& Lockman, F. J. 1990, ARA\&A, 28, 215

Eracleous, M., \& Horne, K. 1994, ApJ, 433, 313

Eisenbart, S., Beuermann, K., Reinsch, K., \& Gänsicke, B. T. 2002, A\&A, 382, 984

Ezuka, H., \& Ishida, M. 1999, ApJS, 120, 277

Fujimoto, R., \& Ishida, M. 1997, ApJ, 474, 774

Haswell, C., Patterson, J., Thorstensen, J. R., Hellier, C., et al. 1997, ApJ, 476, 847

Hellier, C., Mason, K. O., Rosen, S. R., \& Cordova, F. 1987, MNRAS, 228, 463

Hellier, C., Beardmore, A. P., \& Buckley, D. A. H. 1998, MNRAS, 29, 851

Ishioka, R., Kato, T., Uemura, M., et al. 2002, PASJ, 54, 581

Jansen, F., Lumb, D., Altieri, B., et al. 2001, A\&A, 365, L1

Kemp, J., Patterson, J., Thorstensen, J. R., et al. 2002, PASP, 114, 623

King, A. R., \& Lasota, J. P. 1991, ApJ, 378, 674

Mason, K. O., Breeveld, A., Much, R., et al. 2001, A\&A, 365, L36

Matt, G., de Martino, D., Gänsicke, B. T., et al. 2000, A\&A, 358, 177

Mauche, C. W., Liedahl, D. A., \& Fournier, K. B. 2001, ApJ, 560, 992

Mukai, K., Ishida, M., \& Osborne, J. P. 1994, PASJ, 46, L87

Mukai, K., Kinkhabwala, A., Peterson, J. R., et al. 2003, ApJ, 586, L77

Nauenberg, M. 1972, ApJ, 175, 417

Norton, A. J., Beardmore, A. P., \& Taylor, P. 1996, MNRAS, 280, 937

Norton, A.J., Wynn, G.A., \& Somerscales, R.V. 2004, ApJ, 614, 349

Paczynski, B. 1971, ARA\&A, 21, 1

Patterson, J. 1994, PASP, 106, 209

Patterson, J. 1998, PASP, 110, 1132

Porquet, D., \& Dubau, J. 2000, A\&AS, 143, 495

Porquet, D., Mewe, R., Dubau, J., et al. 2001, A\&A, 376, 1113

Roberts, D. H., Lehar, J., \& Dreher, J. W. 1987, AJ, 93, 968

Rosen, S. R., Mason, K. O., \& Cordova, F. A. 1988, MNRAS, 231, 549

Strüder, L., Briel, U., Dennerl, K., et al. 2001, A\&A, 365, L18

Tovmassian, G. H., Greiner, J., Kroll, P., et al. 1998, A\&A, 335, 227

Turner, M. J. L., Abbey, A., Arnaud, M., et al. 2001, A\&A, 365, L27

Warner, B. 1986, MNRAS, 219, 347

Warner, B. 1995, Cataclsymic Variables (Cambridge: Cambridge Univ. Press)

Welsh, W. F., \& Martell, P. J. 1996, MNRAS, 282, 739 